Georgia State University

ScholarWorks @ Georgia State University

$7-2015$

\title{
School Engagement among LGBTQ High School Students: The Roles of Safe Adults and Gay-Straight Alliance Characteristics
}

\author{
Kristie L. Seelman \\ Georgia State University, kseelman@gsu.edu \\ Nicholas Forge \\ Georgia State University, nforge1@gsu.edu \\ N. Eugene Walls \\ University of Denver, eugene.walls@du.edu \\ Nadine Bridges \\ Rainbow Alley, The GLBT Community Center of Colorado, nbridges@glbtcolorado.org
}

Follow this and additional works at: https://scholarworks.gsu.edu/ssw_facpub

Part of the Social Work Commons

\section{Recommended Citation}

Seelman, Kristie L.; Forge, Nicholas; Walls, N. Eugene; and Bridges, Nadine, "School Engagement among LGBTQ High School Students: The Roles of Safe Adults and Gay-Straight Alliance Characteristics" (2015). SW Publications. 60.

https://scholarworks.gsu.edu/ssw_facpub/60

This Article is brought to you for free and open access by the School of Social Work at ScholarWorks @ Georgia State University. It has been accepted for inclusion in SW Publications by an authorized administrator of ScholarWorks @ Georgia State University. For more information, please contact scholarworks@gsu.edu. 
School Engagement among LGBTQ High School Students: The Roles of Safe Adults and Gay-Straight Alliance Characteristics

Kristie L. Seelman ${ }^{\mathrm{a},{ }^{*}}$, Nicholas Forge ${ }^{\mathrm{a}}$, N. Eugene Walls ${ }^{\mathrm{b}}$, Nadine Bridges ${ }^{\mathrm{c}}$

a School of Social Work, Andrew Young School of Policy Studies, Georgia State University, PO Box 3995, Atlanta, GA 30302-3995 United States

${ }^{\mathrm{b}}$ Graduate School of Social Work, University of Denver, 2148 South High Street, Denver, CO 80208-7100 United States

${ }^{\mathrm{c}}$ Rainbow Alley, The GLBT Community Center of Colorado, 1301 E Colfax, Denver, CO 80218 United States

This is the Author's Accepted Manuscript version of the article published by Elsevier in Children \& Youth Services Review on July 30, 2015, available online:

http://dx.doi.org/10.1016/j.childyouth.2015.07.021

(C) 2015. This manuscript version is made available under the CC-BY-NC-ND 4.0 license http://creativecommons.org/licenses/by-nc-nd/4.0/

* Corresponding author at: School of Social Work, Andrew Young School of Policy Studies, Georgia State University, PO Box 3995, Atlanta, GA 30302-3995, United States. Tel.: +1 4044131071

E-mail address: kseelman@gsu.edu (K.L. Seelman). 


\section{Highlights}

- Examines predictors of student school engagement among LGBTQ high school students.

- The more types of safe adults at school, the greater the engagement of LGBTQ youth.

- Presence of a Gay-Straight Alliance (GSA) did not predict student school engagement.

- However, GSA size, visibility, activity and perceived support predict engagement.

- Personal involvement in a GSA did not predict student school engagement. 


\begin{abstract}
Student school engagement, or the person-environment fit between a student and the student's school, is a construct that has received increasing attention in the school psychology literature in recent years. However, little research has examined this construct among lesbian, gay, bisexual, transgender, queer and questioning (LGBTQ) students or analyzed whether factors such as access to safe adults, the presence of a Gay-Straight Alliance (GSA), characteristics of a GSA, or personal involvement in a GSA may connect to engagement. The current study used sequential multiple regression to examine data from a sample of LGBTQ high school students $(N=152)$ from Colorado and found that the greater the number of types of safe adults that a student has access to at school, the higher the student's school engagement. GSA presence was not significantly associated with student school engagement. However, among those students whose school had a GSA $(N=91)$, the larger, more active, more visible, and more supported a GSA was perceived to be, the more these students were engaged at school. Personal involvement in a GSA did not predict student school engagement. This article discusses implications for school-based practitioners and future research.
\end{abstract}

\title{
Keywords
}

LGBTQ youth; high school; school engagement; Gay-Straight Alliance; social support 


\section{Introduction}

Student school engagement, or the person-environment fit between a student and the student's school, is a construct that has received increasing attention in the school psychology literature in recent years (Hazel, Vazirabadi, Albanes, \& Gallagher, 2014; Hazel, Vazirabadi, \& Gallagher, 2013). Student school engagement is a modifiable factor that can predict student academic outcomes such as grades, truancy, and dropping out, as well as nonacademic outcomes such as depression, substance abuse, and delinquency (Fredricks, Blumenfeld, Paris, 2004; Hazel et al., 2013; Lam et al., 2014; Li et al., 2011; Wang \& Fredricks, 2014; Wang \& Peck, 2013). Engagement is theorized to act as a link between school contextual factors and school performance for youth and young adults (Lam et al., 2014). However, little research has examined this construct among lesbian, gay, bisexual, transgender, queer and questioning (LGBTQ) students, a subpopulation that faces increased risk factors (such as harassment, bullying, and a hostile school climate) that may affect academic performance and behaviors at school as well as mental health outcomes.

Additionally, while researchers have previously examined school contextual factors - such as the presence of safe adults or a Gay-Straight Alliance (GSA) - in relation to academic and psychosocial outcomes for LGBTQ students, there is a dearth of research examining how such contextual factors may connect to student school engagement for this population. Further, most studies looking at outcomes for LGBTQ youth have measured GSAs in terms of simple presence, ignoring contextual details such as the GSA's size, level of activity, visibility, or degree of support within the school.

Using a sample of LGBTQ high school students from Colorado, the present study addresses these gaps in the literature, examining whether access to safe adults at school, presence of a GSA, characteristics of GSAs, and one's personal involvement in a GSA predict student school engagement. This paper will first provide an overview of the evidence 
base related to high school settings for LGBTQ youth, the roles of safe adults and GSAs, and student school engagement. This will be followed by a description of the present study's methods and results. The paper concludes with a discussion of findings, limitations, and implications for practitioners working within school settings.

\subsection{High School Settings for LGBTQ Youth}

Adolescence is a key period of development for LGBTQ youth, as many individuals in the U.S. begin to develop a sense of their sexuality and/or gender identity during this time of their lives. As reported by the Institute of Medicine (IOM, 2011), early research on LGBTQ young people has indicated that "coming out" during this time period can present many challenges due to the prevalence of societal homophobia and transphobia and their negative effects on adolescents. Since many youth go through adolescence and the associated developmental tasks while in high school, such settings are a key place of socialization and exposure to ecological factors that may affect the development of LGBTQ youth (Pearson, Muller, \& Wilkinson, 2007).

Building from Bronfenbrenner's ecological perspective, Kosciw, Greytak, and Diaz (2009) assert that LGBTQ youths' feelings of safety or risks for victimization at school not only connect to the individual identities that they hold, but are also affected by factors within the larger social environment, including their school. These environmental factors can create differential experiences for sexual and gender minority students compared to heterosexual, cisgender ${ }^{1}$ students (Kosciw et al., 2009). The IOM (2011) points to how the research base about LGBTQ youth has focused on schools as a key setting of conflict and victimization for this population. A growing body of literature has indicated that middle school and high school climates are often hostile and unsafe for LGBTQ youth, with frequent occurrences of

\footnotetext{
${ }^{1}$ Cisgender is an identity term for those whose gender identity matches cultural expectations for their sex assigned at birth (i.e., they are neither transgender nor gender non-conforming).
} 
harassment, discrimination, and violence (see IOM, 2011 and Kosciw et al., 2009 for a review of related research). Some evidence suggests that the school climate may be particularly difficult for transgender and gender non-conforming youth, with greater likelihood of experiencing assault, harassment, feeling unsafe, and missing days of school due to fear compared to LGB youth (Kosciw, Greytak, Bartkiewicz, Boesen, \& Palmer, 2012; Kosciw, Greytak, Bartkiewicz, Boesen, \& Palmer, 2014). Such risks within the school climate can deeply impact the interaction between individual students and the school setting, producing difficulties for LGBTQ youth that extend into adulthood (Pearson et al., 2007). LGBTQ students who experience victimization in school are more likely to have lower selfesteem, weaker grades, and a greater number of missed days of school, and they are at increased risk for suicide (Birkett, Russell, \& Corliss, 2014; Goodenow, Szalacha, \& Westheimer, 2006; Kosciw et al., 2014; Kosciw, Palmer, Kull, \& Greytak, 2013).

As Kosciw and colleagues (2013) have stated, “A central challenge for educators and safe school advocates is how to identify and design supportive school climates that promote the positive development of LGBT and all students" (pp. 46-47). Research has begun to indicate a number of school-level factors that can counteract heterosexism and cisgenderism ${ }^{2}$ within schools and support the psychological and physical well-being and academic success of LGBTQ students. Examples include nondiscrimination and anti-bullying policies inclusive of sexual orientation and gender identity, LGBTQ-inclusive curricula, changes to the physical environment of the school (e.g., gender-inclusive bathroom and locker room options), having a GSA at school, and having safe staff at school who students can talk to about their sexual orientation and/or gender identity (Chesir-Teran, 2003; Chesir-Teran \& Hughes, 2009; Diaz, Kosciw, \& Greytak, 2010; Kosciw et al., 2013; Sausa, 2005; Walls, Kane, \& Wisneski,

\footnotetext{
${ }^{2}$ Building from the conceptual work of Chesir-Teran (2003), cisgenderism is the systematic process of privileging cisgender (non-transgender) identities relative to transgender and gender-nonconforming identities, based on the assumption that being cisgender is normal and ideal (Seelman, 2013).
} 
2010). This paper will be taking a closer look at what the literature has to say about two of these factors relevant to the present study: access to safe adults at school, and the presence of GSAs.

\subsection{The Role of Safe Adults at School for LGBTQ Students}

As previously detailed, school harassment and violence are commonplace for LGBTQ students in the U.S. A national survey conducted by the Gay, Lesbian and Straight Education Network (GLSEN) found that while three quarters of LGBT students had been verbally harassed in their school, over half (57\%) had failed to report the incident to school staff, mostly due to their not believing that any action would be taken (Kosciw et al., 2014). If harassment and victimization are not directly addressed, they can escalate into physical and sexual violence (Holmes \& Cahill, 2004). Yet, school staff do not necessarily respond to reports of harassment and violence. In their study, GLSEN found that $62 \%$ of LGBT students who had reported such experiences were met with no response or action taken on their behalf by school staff (Kosciw, et al., 2014).

Having access to a safe adult—whether a teacher, nurse, counselor, or principal-serves as an important component in the creation of a safe school for LGBTQ students (Kosciw et al., 2014). Schools with supportive adults and staff help to create an environment where students feel more connected to their education, have a greater sense of school belonging, are more likely to attend activities of a GSA, and are less likely to experience victimization or miss school due to feeling unsafe (Diaz, Kosciw, \& Greytak, 2010; Kosciw et al., 2013, 2014; Murdock \& Bolch, 2005; Seelman, Walls, Hazel, \& Wisneski, 2012). Such adults can have a role in providing safety by preventing harassment and violence. One study found that LGB students who could identify an adult in school they could talk to were about one third more likely to report being threatened or victimized at school compared to those without such an adult (Goodenow et al., 2006). For transgender students, having a connection 
to an adult in school is positively correlated with feeling safe in school, and supportive adults play an important role when navigating the school environment if the student is transitioning (McGuire, Anderson, Toomey, \& Russell, 2010).

Research suggests ways that particular types of adults at schools-such as teachers (Murdock \& Bolch, 2005) or school psychologists (Murphy, 2012)—can provide critical support to LGBTQ students. In their national study, Kosciw et al. (2014) examined students' experiences approaching different types of adults at school, with teachers and school-based mental health professionals (counselors, social workers, etc.) being among the adults approached most frequently and with the greatest level of comfort. Over half of this sample said they would be comfortable talking with a teacher or school-based mental health professional about LGBT issues, while only about one out of four would be comfortable approaching a principal or a librarian (Kosciw et al., 2014). While we did not find other studies that specifically looked at the relationship between number of types of safe adults and outcomes, the knowledge base suggests that knowing safe adults across multiple spheres of the school environment would provide the greatest level of support for LGBTQ students.

\subsection{Gay-Straight Alliances and LGBTQ Students}

The emerging body of scholarship on the relationship between GSAs and psychosocial outcomes for LGBTQ youth and young adults is mixed depending upon the type of outcome examined and whether one is studying presence of GSAs, membership in them, or school contextual factors that may influence the impact of GSAs. Presence of a GSA in a school or college tends to be associated with more positive outcomes for LGBTQ youth than actual membership or involvement in a GSA, although some mixed results do exist. Recent scholarship has started to examine contextual factors about the GSAs themselves and has added to a more nuanced understanding of these relationships. 
Research indicates that having a GSA at school positively correlates with LGBTQ students' comfort with gender expression (Walls, Wisneski, \& Kane, 2013), greater levels of being “out” (Heck, Lindquist, Stewart, Brennan, \& Cochran, 2013), and greater self-esteem (Toomey et al., 2011). Findings are, however, mixed on other mental health outcomes, with some associations with lower levels of depression (Heck, Flentje, \& Cochran, 2013; Toomey et al., 2011) and lower levels of general psychological distress (Heck, Flentje, et al., 2013), but at least one study finding no differences in mental health outcomes (Heck, 2014). Relatedly, most studies have found a positive relationship with lower levels of suicidality (Poteat, Sinclair, DiGiovanni, Koenig, \& Russell, 2013; Walls, Freedenthal, \& Wisneski, 2008), but at least one study found no significant relationship with lifetime suicide attempts (Toomey et al., 2011).

Examining the relationship of GSA presence and victimization in schools, some studies have found no relationship (Poteat et al., 2013; Walls et al., 2010), while others have found a statistically significant relationship (Diaz et al., 2010; Greytak, Kosciw, \& Boesen, 2013; Heck, Flentje et al., 2013). With the exception of Poteat et al. (2013) and Diaz et al. (2010), most scholarship has confirmed a relationship between GSA presence and connections to school, whether measured as school belonging, decreased isolation, ability to identify safe adults, increased comfort and openness, or increased engagement (Fischer, 2011; Garcia-Alonso, 2004; Heck, Flentje, et al., 2013; Quasha, 2011; St. John et al., 2014; Toomey, McGuire, \& Russell, 2012; Walls et al., 2010). A related consistent finding is the relationship between GSA presence and decreased truancy (Greytak et al., 2013; Poteat et al., 2013; Walls et al., 2010). A final area of mixed results is the relationship between GSA presence and school performance or educational attainment. Walls and colleagues (2010; see also Seelman et al., 2012) found relationships with both grade point average and drop out status, but other studies have found no relationship (Poteat et al., 2013; Toomey et al., 2011). 
Fewer studies have examined the relationship between membership or participation in GSAs and psychosocial outcomes, but what does exist has provided an inconclusive picture. On the positive side, findings have suggested a relationship between GSA participation and decreased suicidality (Walls et al., 2013), more positive feelings about one's sexual orientation (Hermann, 2010; Lee, 2002), better school and personal relationships (Lee, 2002), greater school connectedness (Diaz et al., 2010), and greater social maturity (Tamayo, 2008). Findings related to safety and victimization are mixed, with one study finding no relationship (Walls et al., 2010) and another finding a connection between involvement in a GSA and higher levels of victimization (Diaz et al., 2010). Finally, the results of studies looking at academic achievement and performance have been mixed (Hansen, 2009; Lee, 2002; Toomey et al., 2011; Walls et al., 2010). However, the most recent scholarship has suggested that GSA presence may matter more than personal involvement for academic and health outcomes (Toomey et al., 2011; Walls et al., 2010).

Some research has attempted to move beyond a dichotomous understanding of GSAs (i.e., present or not; member or not) and examined contextual factors such as GSA effectiveness, length of time of existence of the GSA, resistance to GSA development, geographical location/region, and administrative support. Contextual factors have been shown to be important in student decisions whether or not to join or participate in GSAs (Heck, Lindquist, et al., 2013), faculty decisions on involvement as GSA sponsors (Graybill, 2011), responsiveness to and frequency of heterosexist remarks in schools (Fischer, 2011), decreased levels of depression (Toomey et al., 2011), greater sense of belonging (Fischer, 2011), increased levels of college-level educational attainment (Toomey et al., 2011), and resistance to the development of GSAs (Mayo, 2008). Some of the mixed findings of the relationships between GSAs and psychosocial outcomes may be a product of methodological 
failings to take into account contextual factors such as these (Poteat et al., 2014), indicating a need for research on GSA characteristics.

Next, we briefly review literature related to the theoretical framework of interestschool engagement theory — as it relates to LGBTQ high school students.

\subsection{School Engagement Theory and LGBTQ Students}

Although there are a number of different approaches and conceptualizations of the construct known as student engagement or school engagement (see Fredricks et al., 2004), we are using an understanding of student school engagement based on the work of Hazel and colleagues (Hazel et al., 2013; Hazel et al., 2014) that defines this concept as a student's assessment of the person-environment fit between oneself and one's school. In this conceptualization, student school engagement encompasses three domains—aspirations, belonging, and productivity. Aspirations encompass a student's commitment to school, based on how worthwhile the student sees school as being for future goals (Hazel et al., 2014). Belonging reflects a student's congruence with school values and norms and feelings of membership within the school. Finally, productivity is one's demonstration of pro-school behaviors, such as doing schoolwork, engaging in tasks that make learning most effective, and connecting with family and other resources to help one achieve success at school (Hazel et al., 2014). Student school engagement is something that is modifiable (Fredricks et al., 2004; Hazel et al., 2013) — that is, the person-environment fit can be affected by various factors in the student's ecological systems.

Lam et al. (2014) have described student engagement as "a psychological process that mediates the effects of the contextual antecedents on student outcomes" (p. 215). In other words, this construct helps us better understand the link between setting-level factors (such as classroom structure and relationships with teachers) and academic outcomes. Such information is useful to capture for LGBTQ students who often face a difficult school 
environment. School engagement has been demonstrated to relate to academic achievement (Fredricks et al., 2004), and this finding has held in an international study of students in grades 7-9 across 12 different countries (Lam et al., 2014). Student school engagement has previously been shown to correlate with better attendance, lower likelihood of failing math or language arts, and lower risk of suspension among $8^{\text {th }}$ graders (Hazel et al., 2013). In a study using latent profile analysis (Wang \& Peck, 2013), high school students classified as either "highly engaged" or "emotionally disengaged" (had high cognitive engagement and moderate behavioral engagement, but low emotional engagement) had the highest GPAs. Other studies have documented that greater school engagement—particularly behavioral or emotional subdomains of the construct — predicts a lower likelihood of dropping out of school (Wang \& Fredricks, 2014; Wang \& Peck, 2013) and this has held true for students of various ethnicities (Fredricks et al., 2004). Wang and Peck (2013) found that students classified as "highly engaged" had the greatest likelihood of enrolling in college. School engagement also appears to have a relationship to non-academic indicators, such as decreased risk for depression (Wang \& Peck, 2013), substance use, and delinquent behaviors (Li et al., 2011; Wang \& Fredricks, 2014).

There is evidence that some features of the school environment can be antecedents of student school engagement. For example, in a review of the literature, Fredricks et al. (2004) detail how being in a small school or having students involved in school policy development can predict engagement. Li et al. (2014) call for more research investigating the school and classroom characteristics that might predict student school engagement.

Many studies on school engagement have focused primarily on White middle class students, and there is a need for further studying differences that may occur in engagement according to one's age, race, ethnicity, and other individual or cultural differences and how students from various backgrounds are affected by changes in the school context (Fredricks et 
al., 2004). There has been some evidence that engagement may differ by such individual differences. In a longitudinal study of a cohort of middle and high school students in the eastern U.S., Wang and Fredricks (2014) found that, in $7^{\text {th }}$ grade, girls had higher scores on behavioral, emotional, and cognitive school engagement compared to boys. White students had lower emotional engagement than African American students in $7^{\text {th }}$ grade, and those with a higher socioeconomic status (SES) had significantly higher cognitive and emotional school engagement scores compared to students with lower SES (Wang \& Fredricks, 2014). Another study (Estell \& Perdue, 2013) indicated that girls and students without disabilities had significant greater behavioral and affective school engagement in fifth grade than boys and students with disabilities.

Few studies have focused on student school engagement among LGBTQ adolescents. In a study of 315 sexual minority youth, Seelman et al. (2012) found that higher student school engagement among high school and college students predicted a higher GPA, and this relationship was most pronounced among students whose school had a GSA. Additionally, findings indicated an interaction between student school engagement and feeling unsafe or afraid at school in predicting fear-based truancy: higher levels of student school engagement predicted less fear-based truancy among youth and young adults who felt unsafe or afraid at school most often (Seelman et al., 2012).

In a project analyzing data from the National Longitudinal Study of Adolescent Health and the Adolescent Health and Academic Achievement study, Pearson, Muller, and Wilkinson (2007) compared adolescents with same-sex and different-sex attractions and found that youth who had same-sex attractions in middle school were more likely to have lower grades in high school and more likely to fail a class. While the data did not include a measure of student school engagement, there were some items related to integration within one's school, including feeling close to others, being attached to teachers and disengaging 
from school. Those who had same-sex attractions in middle school were significantly less integrated or attached to the school, had greater disengagement, and were less attached to their teachers than youth with different-sex attractions. Boys showed the greatest difference in school performance between these two attraction groups, and this difference remained even when controlling for substance use and emotional distress, indicating that integration in the school plays an important role for these boys' academic outcomes (Pearson et al., 2007).

In a study of 101 LGB youth, Murdock and Bolch (2005) found that LGB exclusion in the high school environment was significantly associated with a lower sense of school belonging, even after controlling for teacher support and personal victimization at school. While sense of belonging (feeling valued) differs from school engagement, belonging is one of the three subdomains of Hazel et al.'s (2014) conceptualization of student school engagement and thus plays an important role in the construct. Diaz et al. (2010), who also examined the construct of school belonging (calling it "school connectedness") among LGBT students, found that having a comprehensive anti-harassment policy at school that included LGBT people was associated with greater school connectedness.

Among the existing studies of student school engagement or school belonging among LGBT youth, very few include any subgroup analysis that examines differences by demographics such as race, ethnicity, gender identity, or social class. Such differences are likely important to student school engagement, as students who are in the minority in their school across multiple identities likely experiences less of a fit with the school and are therefore less engaged.

\subsection{Gaps in the Literature}

While the literature suggests that LGBTQ students benefit from having safe adults at school, and there is mixed research about psychosocial and academic outcomes of having a GSA at one's school, there is scant research about how contextual factors-such as GSA size, 
visibility, activity, or level of verbal support for a GSA in school-impact LGBTQ students. Such research could help us understand other dimensions of GSAs (beyond a simple presence/absence measure) that may impact LGBTQ students. Additionally, there have been mixed findings about whether participating in a GSA predicts various psychosocial and academic outcomes for LGBTQ youth, and more research can help explore the degree to which personal involvement matters for individual students.

Student school engagement has received increasing attention from researchers in recent years in terms of how it relates to students' academic achievement, behavior at school, and likelihood of graduating. Yet, little research has examined this construct among LGBTQ students, a subpopulation that faces increased risk factors (such as harassment, bullying, and a hostile school climate) that may affect academic outcomes and behaviors at school. Even fewer studies of LGBTQ youth have included a breakdown within their analyses by race, gender identity, or social class when it comes to engagement. While there have been some key studies looking at the connection between access to safe adults, GSA presence and participation, and sense of school belonging for LGBTQ students, there is little to no research investigating whether such factors, or the characteristics of a GSA, predict the broader construct of student school engagement. The present study addresses these gaps in the knowledge base.

\subsection{Hypotheses}

Based on the review of the literature, this study tests the following hypotheses, controlling for demographic variables: (a) the greater the number of types of safe adults available at school with whom one could openly talk to about sexual orientation or gender identity, the higher the student's school engagement score will be; (b) the presence of a GSA in high school will be associated with higher student school engagement scores among LGBTQ students; (c) for LGBTQ youth whose school has a GSA, the characteristics of that 
GSA (size, visibility, verbal support, and activity) will predict student school engagement: the more strong, visible, well-supported, and active a GSA is perceived to be, the more LGBTQ students will be engaged at school; and (d) for LGBTQ youth whose school has a GSA, personal involvement in a GSA will $\underline{\text { not }}$ be a statistically significant predictor of student school engagement (null hypothesis). This final hypothesis, while perhaps counterintuitive to some readers, is based upon recent studies suggesting that while GSA involvement connects to some mental health outcomes (Hermann, 2010; Lee, 2002; Toomey et al., 2011; Walls et al., 2013) involvement might not be as important for academic outcomes as whether or not there is a GSA present at school in the first place (Toomey et al., 2011; Walls et al., 2010). We build our hypothesis from this evidence, and also investigate whether the particular characteristics of a GSA have a stronger association with student engagement than one's personal decision to be involved.

\section{Methods}

\subsection{Sample Recruitment and Characteristics}

The present sample was recruited through an LGBTQ community organization in Colorado. Participants in the study were part of a larger sample of LGBTQ youth and young adults who participated in an annual programmatic survey and needs assessment for the youth program within this organization or who attended social events sponsored by the organization and its partner organizations. The original sampling frame was designed to capture youth and young adults ages 13 to 25 who identified as LGBTQ or as other sexual or gender minority identities (such as pansexual, asexual, or genderqueer). Participants were recruited through two methods: (a) direct contact by staff at one of three LGBTQ youth drop-in centers in Colorado partnering with the lead organization on survey distribution, or (b) online advertisement of the survey on the website of the lead organization. The survey was conducted in 2010 and was only available in an online format. Staff informed the youth and 
young adults that participation was optional and a decision about whether to participate would not affect the services they received at any of the partnering organizations.

Respondents were required to indicate electronic consent on the survey website before beginning the survey. Participants were eligible to enter a lottery drawing for gift certificates following participation in the survey. All survey data were anonymous (no individually identifying information was collected).

This study was approved by the IRB of both universities affiliated with the authors as secondary data analysis of anonymous data. None of the authors were involved in sample recruitment or data collection.

\subsection{Measures}

The survey was estimated to take approximately 20 minutes to complete and covered topics ranging from school experiences (truancy, bullying, harassment, and assault) to substance use, non-suicidal self-injury, and use of local LGBTQ organizational services. A number of survey questions were modeled on the National Youth Risk Behavior Surveillance Survey (CDC, 2007) and the National School Climate Survey conducted by the Gay, Lesbian, and Straight Education Network (Kosciw, Diaz, \& Greytak, 2008). The research team selected variables of interest from the survey to use for present analyses, based on a review of theories of student school engagement and gaps in the literature related to LGBTQ students.

\subsubsection{Demographic and control variables}

There is evidence that school engagement may differ by demographic characteristics such as socioeconomic status, race, and gender, as these identities often interact with one's "fit" with a school environment, particularly in circumstances where one's identity differs from the majority of students and faculty within a school. With LGBT students, there is also evidence that certain subgroups, such as transgender youth and youth of color, experience harassment and assault based upon these other identities in school settings, in addition to 
ways they may be targeted based upon sexual orientation (Kosciw et al., 2011, 2013). This suggests an importance to looking for differences in school engagement across these identities. We therefore examined the data in the present study for differences in student school engagement by demographics including participation in free or reduced-price school lunches, race/ethnicity, age, and gender, but did not find statistically significant differences, perhaps due to a smaller sample size and lack of information about the demographic makeup of students' high schools. Nonetheless, we included several demographic variables as controls in our multiple regression model, including: a White/non-White dummy variable for race, current or previous participation in free- or reduced-price school lunch (a dichotomous variable acting as a proxy for low household income), current age, and transgender identity (a dichotomous variable indicating transgender or cisgender identity). We chose to dichotomize the gender identity variable in order to more clearly highlight potential differences between transgender and cisgender students, considering the differing rates of harassment and violence that appear to impact these groups in other studies (Kosciw et al., 2011). However, including the gender identity variable meant that we did not include sexual orientation due to a high degree of multicollinearity between these dimensions.

\subsubsection{Independent Variables}

The primary variables of interest in predicting student school engagement focused on school climate and supports, GSA presence and characteristics, and the student's level of participation in a GSA. The first variable was the number of types of safe adults LGBTQ students knew in high school, measured by the question "Are there adults at school you can talk openly to about your sexual orientation or gender identity?" For students not currently in high school, this question was phrased to ask about their last year in school. Participants responded either yes or no for the following types of adults: Teacher(s), Librarian(s), Principal(s), Guidance Counselor(s), and School Social Worker(s) or Counselor(s). There 
was also an Other response option where participants could write-in another type of supportive adult in school. For our analyses, we chose to form a summative measure of the number of types of safe adults that students knew in high school, as we anticipated that the more types of safe adults students knew, the more they would be engaged in school, regardless of role of that adult. The survey did not collect data about an actual count of how many safe adults students knew in each category (e.g., the number of safe teachers), so we were unable to measure the total number of safe adults.

Students were asked whether their school had a GSA. For our analyses, GSA presence was treated as a dichotomous variable (Yes, or No/Don't know). The survey also included a series of questions inquiring about the characteristics of the GSA in one's school; these questions were part of a skip pattern so that only students whose current or most recent school had a GSA answered these questions. The first question asked about the estimated size of the GSA, with response options including Very small (only 2-4 members), Small (5-10 members), Medium (10-25 members, and Large (25 or more members) ${ }^{3}$. The second question asked how active the GSA was, with a 5-point scale ranging from Very Active to Not at all Active. A third question asked how visible the GSA was, with a 5-point response scale ranging from Very visible to Not at all visible. A fourth question asked how most people at school talk about the GSA, with six responses ranging from Very supportive of the GSA to Very hostile toward the GSA.

The final independent variable of interest was level of personal activity in a GSA. This was measured on a 5-point response scale ranging from Not at all active to Very active.

\subsubsection{Dependent Variable}

\footnotetext{
${ }^{3}$ Some of the response options for GSA size had overlapping ranges-both Small \& Medium included the number 10 in their membership range, and both Medium and Large included the number 25. This may have affected the accuracy of this measure, particularly if respondents were answering according to the suggested membership ranges rather than the general descriptors of Very Small, Small, Medium, etc.
} 
Student school engagement was measured using a selection of nine items from the Student School Engagement Measure (SSEM, Hazel et al., 2013) meant to reflect the three domains of the construct - aspirations, belonging, and productivity. Example items included "My school work is important to me," "Kids at school like me," and "When I am in class I pay attention to my teachers." Respondents who had not been in high school for the past year were asked to respond to these items describing their most recent year in high school. Participants could choose from four Likert-type response options, ranging from Strongly Disagree to Strongly Agree. The nine items were added together (with negatively-worded items reverse scored) to compute a final score of student school engagement.

\subsection{Analyses}

Two sequential multiple linear regression models were constructed to test the hypotheses using the Statistical Package for the Social Sciences (SPSS) version 21. From the original data, 153 cases met the criteria for inclusion in the current study. That is, respondents had to be currently in high school or enrolled in high school in the previous year and identify as LGBTQ. One case was dropped that was an outlier on the age variable (age $=22$ ), resulting in a final analytic sample of 152 respondents for the first model. The second model was constructed to look solely at youth whose current or most recent high school had a GSA $(N=91)$. Variables were examined in relation to assumptions of linear regression and patterns of missingness; all predictor variables were missing at random or missing completely at random.

\section{Results}

\subsection{Descriptive Statistics}

Participants ranged in age from 13 to 19 , with an average age of $16.2(\mathrm{SD}=1.25)$ and a median age of 16 . The largest proportion of the sample was White $(64.5 \%, n=111)$, while $15.1 \%(n=26)$ were Hispanic/Latino/a, $14 \%(n=24)$ were biracial/multiracial, and $6.4 \%(n$ 
$=11)$ identified as some other race or ethnicity. Just over half of the sample $(51.2 \%, n=88)$ identified as female, 37.2\% $(n=64)$ identified as male, 4.7\% $(n=8)$ identified as gender variant/genderqueer, $2.9 \%(n=5)$ identified as trans/female-to-male, $2.9 \%(n=5)$ identified as "other" or unsure of their gender identity, and 1.2\% $(n=2)$ identified as trans/male-tofemale. In terms of sexual orientation, 36.3\% $(n=65)$ identified as bisexual, $26.8 \%(n=48)$ identified as gay, $17.9 \%(n=32)$ identified as lesbian, $7.8 \%(n=14)$ were not sure/questioning, 3.9\% $(n=7)$ identified as queer, 6.7\% $(n=12)$ identified as pansexual or some other identity, and $0.6 \%(n=1)$ identified as heterosexual but had a gender identity of transgender or genderqueer.

For educational status, $15.4 \%(n=32)$ were in $9^{\text {th }}$ grade, $19.7 \%(n=41)$ were in $10^{\text {th }}$ grade, $24 \%(n=50)$ were in $11^{\text {th }}$ grade, $29.3 \%(n=61)$ were in $12^{\text {th }}$ grade, $1 \%(n=2)$ were in GED preparation classes, $4.3 \%(n=9)$ were in an alternative high school, $3.8 \%(n=8)$ had finished high school and were not attending college, and 2.4\% $(n=5)$ had dropped out of high school. Those who had graduated or dropped out of school within the past year were asked to share information about school experiences in their most recent year of high school. A substantial minority $(41.2 \%, n=77)$ of the sample had received free or reduced price school lunches at some point in time. While the survey did not ask for the name of the school the youth most recently attended, they were asked which city (Boulder, Colorado Springs, Denver, Ft. Collins, Grand Junction, Pueblo, or other) was closest to where they currently lived. More than half (56.6\%) lived closest to Denver, while 21.1\% said "Other," $9.7 \%$ were closest to Colorado Springs, 6.3\% were closest to Boulder, and 6.3\% said either Ft. Collins, Grand Junction, or Pueblo. Most of those who said "Other" reported other towns in Colorado or suburbs of the larger cities; a few reported locations outside of Colorado, but since they may have attended school in Colorado in the past year and then moved out-of-state, these cases were retained. 
The participants were also asked a series of questions about their school experiences. One question focused on whether there were adults at school that youth could talk to about the young person's sexual orientation or gender identity. More than half $(62.9 \%, n=117)$ said they knew of a teacher they could approach, 19.8\% $(n=36)$ knew of a safe librarian, $25.8 \%(n=47)$ knew of a safe principal, $56.5 \%(n=104)$ knew of a safe guidance counselor, $47.5 \%(n=86)$ knew of a safe school social worker or counselor, and 1.4\% $(n=3)$ listed another type of safe adult at school outside of these categories. In terms of the number of types of safe adults at school that youth had access to, $26.3 \%(n=47)$ knew of no safe adults, $16.2 \%(n=29)$ knew of one type of safe adult, $16.2 \%(n=29)$ knew of two types of safe adults, $16.2 \%(n=29)$ knew of three types of safe adults, $12.8 \%(n=23)$ knew of four types of safe adults, and $12.3 \%(n=22)$ knew of five types of safe adults. The average number of types of safe adults that youth knew at school was $2.10(\mathrm{SD}=1.73)$.

More than half $(56.4 \%, n=106)$ reported that their current or most recent school had a GSA, while $43.6 \%(n=82)$ either did not have a GSA at school or did not know if there was one. Table 1 displays the percentages and frequencies for the questions about GSA characteristics, including GSA size, activity level, visibility, and how supportive most people were when discussing the GSA at school (what we're calling perceived support), as well as level of personal involvement. Among those who had a GSA at school, the mean responses indicated that the average GSA was perceived as Small, Slightly Active, Slightly Visible, and Slightly Supported by most people at school who talked about it. Respondents were typically Slightly Active in the group (although the mode response was Not at all Active).

Several of the GSA variables are theorized to measure similar aspects of GSA presence, such as size, activity, visibility, and perceived support within the school. Each of these four variables had strong correlations with each other, ranging from 0.84 to 0.91 . To 
Table 1

Descriptive statistics about GSA characteristics variables and level of personal involvement in a GSA

\begin{tabular}{|c|c|c|c|c|}
\hline & \multicolumn{2}{|c|}{$\begin{array}{c}\text { School has } \\
\text { a GSA }\end{array}$} & \multicolumn{2}{|c|}{$\begin{array}{c}\text { Including } \\
\text { those without } \\
\text { a GSA } \\
\end{array}$} \\
\hline & $n$ & $\%$ & $n$ & $\%$ \\
\hline \multicolumn{5}{|l|}{ GSA Size } \\
\hline No GSA/Don't know & & & 82 & 43.6 \\
\hline Very small (2-4 members) & 19 & 17.9 & 19 & 10.1 \\
\hline Small (5-10 members) & 48 & 45.3 & 48 & 25.5 \\
\hline Medium (10-25 members) & 27 & 25.5 & 27 & 14.4 \\
\hline Large $(25+$ members $)$ & 12 & 11.3 & 12 & 6.4 \\
\hline \multicolumn{5}{|c|}{ GSA Activity } \\
\hline No GSA/Don't know & & & 82 & 43.6 \\
\hline Not at all active & 9 & 8.5 & 9 & 4.8 \\
\hline Hardly active at all & 24 & 22.6 & 24 & 12.8 \\
\hline Slightly active & 22 & 20.8 & 22 & 11.7 \\
\hline Somewhat active & 46 & 43.4 & 46 & 24.5 \\
\hline Very active & 5 & 4.7 & 5 & 2.7 \\
\hline \multicolumn{5}{|l|}{ GSA Visibility } \\
\hline No GSA/Don't know & & & 82 & 43.6 \\
\hline Not at all visible & 11 & 10.5 & 11 & 5.9 \\
\hline Hardly visible at all & 40 & 38.1 & 40 & 21.4 \\
\hline Slightly visible & 26 & 24.8 & 26 & 13.9 \\
\hline Somewhat visible & 18 & 17.1 & 18 & 9.6 \\
\hline Very visible & 10 & 9.5 & 10 & 5.3 \\
\hline \multicolumn{5}{|l|}{ Perceived Support for GSA } \\
\hline No GSA/Don't know & & & 82 & 43.6 \\
\hline Very hostile & 6 & 5.7 & 6 & 3.2 \\
\hline Somewhat hostile & 6 & 5.7 & 6 & 3.2 \\
\hline Slightly hostile & 20 & 18.9 & 20 & 10.6 \\
\hline Slightly supportive & 43 & 40.6 & 43 & 22.9 \\
\hline Somewhat supportive & 23 & 21.7 & 23 & 12.2 \\
\hline Very supportive & 8 & 7.5 & 8 & 4.3 \\
\hline \multicolumn{5}{|l|}{ Personally Active in GSA } \\
\hline No GSA/Don't know & & & 82 & 43.6 \\
\hline Not at all active & 33 & 31.1 & 33 & 17.6 \\
\hline Hardly active at all & 16 & 15.1 & 16 & 8.5 \\
\hline Slightly active & 11 & 10.4 & 11 & 5.9 \\
\hline Somewhat active & 17 & 16.0 & 17 & 9.0 \\
\hline Very active & 29 & 27.4 & 29 & 15.4 \\
\hline
\end{tabular}


help avoid multicollinearity in the final regression models yet maintain each of these dimensions related to GSA presence, we created a composite scale of GSA characteristics. Each variable was scored so that responses indicating a stronger, positive presence of a GSA (bigger in size, more visible, etc.) received the highest score on the response scales; additionally, since not all items had the same number of responses, GSA size and perceived support were rescaled so that scores were adjusted to be on a scale from 1 to 5 . The Cronbach's alpha for the calculated GSA characteristics scale was 0.76 , which indicates an acceptable level of internal reliability. The mean score on the GSA characteristics composite variable was $12.06(\mathrm{SD}=3.30)$, with a minimum score of 4.06 and a maximum score of 19.09 (the full range of possible scores was from 4 to 20). Because of our interest in investigating the unique contributions of personal involvement in a GSA in predicting student school engagement, we kept this as a separate predictor variable from the other GSA characteristics items.

For the dependent variable, student school engagement, scores were calculated as the sum of all nine items (with possible responses ranging from $1=$ Strongly Disagree to $4=$ Strongly Agree). One item (When I am in class, I often think about other things) was reverse scored. The internal reliability (Cronbach's alpha) for this scale was strong: $a=0.81$ for students currently in high school, and $a=0.75$ for students who had dropped out or graduated from high school but were not currently in college. The summed scores on the student school engagement scale ( $n=186$ ) ranged from 12 to 36 (out of a possible range of 9 to 36), with a mean of $26.41(\mathrm{SD}=4.03)$, a median of 26.50 , and a mode of 26 . These scores indicate that a typical respondent answered the SSEM questions with a response just slightly below Agree. Among students with a GSA at school $(n=103)$, the average score on the student school engagement scale was $26.83(\mathrm{SD}=3.60)$, and scores ranged from 18 to 35 .

\subsection{Inferential Statistics}


Two sequential multiple regression models were constructed to test the four hypotheses; one model, which utilizes both students with and without a GSA at school, examines whether access to safe adults and GSA presence are significant predictors of student school engagement. The second model focuses only on youth who reported that their school had a GSA and examines whether GSA characteristics and personal involvement are associated with engagement. Tables 2 and 3 include correlation information for variables for each of the two models.

Table 2

Correlations for variables used in Model $1(N=152)$

\begin{tabular}{llllllll}
\hline Variables & 1 & 2 & 3 & 4 & 5 & 6 & 7
\end{tabular}

Demographics

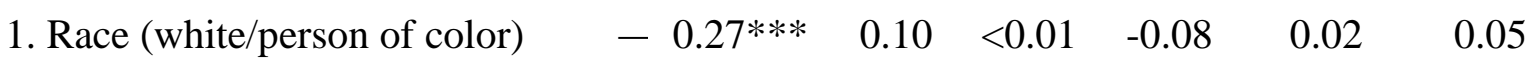

$\begin{array}{lllllll}\text { 2. Free/reduced price lunch } & - & 0.09 & 0.08 & -0.08 & -0.04 & 0.07\end{array}$

3. Age $\quad-\quad \begin{array}{lllll}0.10 & 0.08 & 0.13 & 0.10\end{array}$

4. Gender (transgender/cisgender) $\quad-\quad \begin{array}{llll}-0.12 & 0.12 & -0.07\end{array}$

Predictor variables

5. Safe adults

6. GSA presence

- $\quad 0.12$

Outcome variable

7. Student school engagement

** $p<.01 . * * * p<.001$.

Since missingness for all variables was at random or completely at random and there were not large proportions of missing data, cases with missing data on any of the analysis variables 
Table 3

Correlations for variables used in Model $2(N=91)$

\begin{tabular}{|c|c|c|c|c|c|c|c|}
\hline Variables & 1 & 2 & 3 & 4 & 5 & 6 & 7 \\
\hline \multicolumn{8}{|l|}{ Demographics } \\
\hline 1. Race (white/person of color) & - & $0.33 * *$ & 0.07 & -0.02 & -0.13 & $-0.21 *$ & 0.01 \\
\hline 2. Free/reduced price lunch & & - & 0.07 & 0.08 & 0.03 & -0.09 & 0.05 \\
\hline 3. Age & & & - & $0.24^{*}$ & -0.18 & $-0.20 *$ & $0.22^{*}$ \\
\hline 4. Gender (transgender/cisgender) & & & & - & 0.11 & 0.05 & -0.02 \\
\hline
\end{tabular}

Predictor variables

5. GSA characteristics

$-0.64 * * * 0.21 *$

6. GSA personally active

$-\quad 0.07$

Outcome variable

7. Student school engagement

$* p<.05 . * * p<.01 . * * * p<.001$.

were dropped listwise, leaving a sample of $N=152$ for the first model (all high school students) and $N=91$ for the second model (students at schools with a GSA).

\subsubsection{Safe Adults and GSA Presence}

For the first regression model that included youth at schools either with or without a GSA, the first block of variables included individual demographics (a dummy variable for race, a dummy variable for free/reduced-price lunch participation, age, and a transgender/cisgender dummy variable). The second block included two school-level characteristics (the number of types of safe adults that a student knew, and whether or not the school had a GSA). 
Table 4 includes information about the multiple regression model. For Block 1 of the model, $R$ was not significantly different from zero, $F(4,148)=1.12, p=0.35$, and the adjusted $R^{2}$ was very small $(<0.01)$, indicating that the individual demographics included in the regression model accounted for less than $1 \%$ of the variance in student school engagement among this sample of LGBTQ students. None of the demographic variables had a statistically significant beta coefficient in predicting student school engagement.

Block 2 added the two school variables - number of types of safe adults known at school, and whether the student's school had a GSA. The $R$ for Block 2 was significantly different from zero, $F(6,146)=2.29, p=0.04$. The adjusted $R^{2}=0.05$ indicating that $5 \%$ of the variance in student school engagement was accounted for once safe adults and GSA presence were added to the model. The demographic variables remained not significant. After controlling for demographics and GSA presence, the number of types of safe adults that a LGBTQ student knew in high school was a statistically significant predictor of student school engagement, $\mathrm{B}=0.53, p<.01$. For each additional type of safe adult that a young person knew in high school, that student's engagement score was predicted to increase by 0.53 . This means that, compared to a student who did not know any safe adults, a student who knew of adults in five different types of roles who were safe to talk to about sexual orientation or gender identity was predicted to have a student school engagement score that was 2.65 points higher. After controlling for demographics and access to safe adults at school, GSA presence alone was not a statistically significant predictor of student school engagement among this sample.

\subsubsection{GSA Characteristics and Personal Involvement}

The second regression model (see Table 5) focused only on youth whose school had a GSA $(N=91)$. The first block of this model included the same demographic variables used in 
Table 4

Sequential multiple regression model predicting student school engagement using number of types of safe adults and presence of a GSA $(N=152)$

\begin{tabular}{|c|c|c|c|}
\hline Predictor Variables & $\begin{array}{c}\text { Block 1 B } \\
\text { (s.e.) }\end{array}$ & $\begin{array}{c}\text { Block } 2 \text { B } \\
\text { (s.e.) }\end{array}$ & $\begin{array}{c}\text { Final } \\
\text { Model } \beta\end{array}$ \\
\hline \multicolumn{4}{|l|}{ Demographics } \\
\hline Race (white/person of color) & $\begin{array}{c}0.31 \\
(0.69)\end{array}$ & $\begin{array}{c}0.28 \\
(0.68)\end{array}$ & 0.04 \\
\hline Free/reduced price lunch & $\begin{array}{c}0.92 \\
(0.68)\end{array}$ & $\begin{array}{c}0.97 \\
(0.67)\end{array}$ & 0.12 \\
\hline Age & $\begin{array}{c}0.26 \\
(0.26)\end{array}$ & $\begin{array}{c}0.15 \\
(0.25)\end{array}$ & 0.05 \\
\hline Gender (transgender/cisgender) & $\begin{array}{l}-1.15 \\
(1.04)\end{array}$ & $\begin{array}{l}-1.40 \\
(1.04)\end{array}$ & -0.11 \\
\hline \multicolumn{4}{|l|}{ School variables } \\
\hline Safe adults (\# types known) & & $\begin{array}{c}0.53 * * \\
(0.19)\end{array}$ & 0.24 \\
\hline GSA presence & & $\begin{array}{c}0.12 \\
(0.68)\end{array}$ & 0.02 \\
\hline \multicolumn{4}{|l|}{ Model Results } \\
\hline$F$-value & 1.12 & $2.29 *$ & \\
\hline$R$ & .17 & .29 & \\
\hline$R^{2}$ & .03 & .09 & \\
\hline Adjusted $R^{2}$ & $<.01$ & .05 & \\
\hline
\end{tabular}

Model 1. The second block included GSA characteristics and personal involvement in a GSA to see if these variables would be significantly associated with student school engagement. The $R$ for Block 1 did not significantly differ from zero, $F(4,87)=1.32, p=0.27$, and the adjusted $R^{2}$ was again very small (0.01), indicating that the individual demographics accounted for less than $1 \%$ of the variance in student school engagement among this sample of LGBTQ students who attended a high school that had a GSA. Only age had a statistically significant relationship to school engagement, $\mathrm{B}=0.68, p<.05$. For every one year increase in age, student school engagement was predicted to increase by 0.68 points among students whose school had a GSA. 
Table 5

Sequential multiple regression model predicting student school engagement using GSA characteristics and personal involvement in a GSA $(N=91)$

\begin{tabular}{|c|c|c|c|}
\hline Predictor Variables & $\begin{array}{c}\text { Block } 1 \text { B } \\
\text { (s.e.) }\end{array}$ & $\begin{array}{c}\text { Block } 2 \text { B } \\
\text { (s.e.) }\end{array}$ & $\begin{array}{c}\text { Final } \\
\text { Model } \beta\end{array}$ \\
\hline \multicolumn{4}{|l|}{ Demographics } \\
\hline Race (white/person of color) & $\begin{array}{l}-0.34 \\
(0.89)\end{array}$ & $\begin{array}{c}0.14 \\
(0.86)\end{array}$ & -0.02 \\
\hline Free/reduced price lunch & $\begin{array}{c}0.78 \\
(0.87)\end{array}$ & $\begin{array}{c}0.49 \\
(0.85)\end{array}$ & 0.06 \\
\hline Age & $\begin{array}{l}0.68^{*} \\
(0.33)\end{array}$ & $\begin{array}{l}0.84^{*} \\
(0.32)\end{array}$ & 0.28 \\
\hline Gender (transgender/cisgender) & $\begin{array}{l}-1.05 \\
(1.61)\end{array}$ & $\begin{array}{l}-1.62 \\
(1.56)\end{array}$ & -0.11 \\
\hline \multicolumn{4}{|l|}{ GSA-related variables } \\
\hline GSA characteristics & & $\begin{array}{c}0.33 * * \\
(0.12)\end{array}$ & 0.37 \\
\hline GSA personal activity & & $\begin{array}{l}-0.23 \\
(0.31)\end{array}$ & -0.10 \\
\hline \multicolumn{4}{|l|}{ Model Results } \\
\hline$F$-value & 1.32 & $2.46^{*}$ & \\
\hline$R$ & .24 & .38 & \\
\hline$R^{2}$ & .06 & .15 & \\
\hline Adjusted $R^{2}$ & .01 & .09 & \\
\hline
\end{tabular}

Block 2 added the GSA-related variables: the composite score of GSA characteristics (size, activity, visibility, and perceived support) and personal involvement in a GSA. The $R$ for this Block significantly differed from zero, $F(6,85)=2.46, p=0.03$. The adjusted $R^{2}=.09$, indicating that $9 \%$ of the variance in student school engagement was accounted for by the variables in this model. Age remained the only statistically significant demographic variable $(\mathrm{B}=0.84, p<.05)$. Among LGBTQ high school students who had a GSA at school, the characteristics of one's GSA were a statistically significant predictor of student school engagement, $\mathrm{B}=0.33, p<.01$. For every one point increase in the GSA characteristics scale (meaning an increase in the GSA's visibility, size, activity, and verbal support at school), student school engagement was predicted to increase 0.33 points. This means that someone who scored at the top of the GSA characteristics scale would be predicted to have a student 
school engagement score 5.28 points higher than someone scoring at the bottom of the GSA characteristics scale. Finally, as predicted, personal involvement in a GSA was not a statistically significant predictor of student school engagement $(\mathrm{B}=-0.23, p=0.46)$.

\section{Discussion}

This study addresses a number of gaps in the literature about LGBTQ youth in high school settings, including an analysis of student school engagement among this population and the relationship between having access to safe adults and GSAs with student school engagement. Specifically, this study went beyond looking at simple GSA presence to examine the ways that GSA characteristics (size, activity, visibility, and support within a school) as well as personal involvement in a GSA relate to engagement. Three of our four hypotheses were confirmed through the multiple regression models.

The first hypothesis was that the greater the number of types of safe adults a student perceives to be available at school for discussing sexual orientation or gender identity, the higher the student's school engagement score will be. Our data support this hypothesis—after controlling for the demographic variables and presence of a GSA, the more types of safe adults students knew at school, the higher their student school engagement scores were predicted to be. This finding is in line with previous research indicating the benefits for LGBTQ youth of having safe adults in school, such as feeling more connected to their schooling, having a higher GPA, skipping school less often due to fear (Kosciw, et al., 2014; Seelman et al., 2012) and, for transgender students, feeling safer in school (McGuire et al., 2010).

Such a finding reflects how personal relationships matter for LGBTQ studentsadults who communicate an openness, rather than hostility, about LGBTQ students contribute to the overall high school climate for this population. Having access to a safe adult is not just about increasing a student's comfort; since school engagement is theorized to play an 
important role in mediating the connection between school context and academic outcomes, safe adults could help contribute to the pathway between school engagement and stronger school performance for these students, although this was not examined in the present study. Further, because our study looked at the number of types of safe adults, the evidence here supports the idea that students can benefit from having access to safe adults in a variety of roles - from teachers to social workers, principals, and janitors. In order to encourage and support the presence of safe adults within a school setting, administrators can provide staff with access to safe zone trainings and information about appropriate terminology in working with LGBTQ students, post visual representations of LGBTQ people or safe space signage in the school, and ensure curricula provided by teachers are LGBTQ-inclusive.

Secondly, we hypothesized that GSA presence would be associated with greater school engagement, as a number of previous studies have suggested that having a GSA at school relates to school belonging, decreased isolation, greater ability to identify safe adults, increased comfort and openness, and increased engagement for LGBTQ students (Fischer, 2011; Garcia-Alonso, 2004; Heck, Flentje, et al., 2013; Quasha, 2011; St. John et al., 2014; Toomey et al., 2012; Walls et al., 2010). After controlling for demographics and the number of types of safe adults that students knew, GSA presence was not significantly associated with student school engagement. This is in line with a smaller proportion of recent research (Diaz et al., 2010; Poteat et al., 2013) that has indicated that GSA presence alone does not relate to school belonging. Perhaps presence of a GSA is confounded by exactly what that presence looks like - some high schools may have a GSA that LGBTQ students know about, and yet it may be quite small, inactive, or ineffective at changing anti-LGBTQ hostility within the school culture. In such cases, a GSA may have very little to contribute to an LGBTQ student's feeling of "fit" with the school setting. For that reason, some scholars have recently encouraged a more nuanced look at the characteristics of GSAs — such as visibility, 
size, and level of support - to understand how these factors may matter in terms of student outcomes (Heck et al., 2013; Poteat et al., 2013).

We next looked at engagement among students specifically at schools with GSAs and whether GSA characteristics and personal involvement in a GSA would relate to student school engagement. The only statistically significant demographic variable was age-we found that being older was associated with greater school engagement. This finding suggests that engagement may be lower for students first entering high school compared to those who are older, which may be a reflection of the time it takes students to assess a school's culture and norms, determine how much they "fit" within that culture, and begin to feel welcome. Additionally, one might theorize that students who drop out after $9^{\text {th }}$ or $10^{\text {th }}$ grade, for example, might have persistently lower school engagement, but since they are not in school at older ages, only those with higher engagement scores are captured among the older students.

Our third hypothesis was that, among students whose school has a GSA, the characteristics (size, visibility, verbal support, and activity) of that GSA would predict student school engagement. This hypothesis was confirmed, as results indicate that the more large, visible, well-supported, and active a GSA was perceived to be within a school, the more LGBTQ students were engaged at school. This finding supports other recent studies about contextual factors of a school mattering in relation to the development of GSAs and the impact on LGBTQ students (Fisher, 2011; Konishi, Saewyc, Homma, \& Poon, 2013; Mayo, 2008; Toomey et al., 2011), with the present study filling a particular gap in connection to school engagement.

Thus, the role of GSAs may be more nuanced than simply noting whether they are present or not - it is important to study how a GSA's characteristics matter in relation to the school climate. Our findings indicate that, in relation to student school engagement, it matters whether the GSA engages in regular activities, whether it is visible, whether it attracts a lot of 
students or only a few, and whether people in the school generally talk positively or negatively about the GSA. LGBTQ students who attend a school that has a GSA but the group is very small, nearly invisible, inactive, and/or criticized by peers are less likely to experience the GSA as a boost to engagement. Thus, the characteristics of GSAs offer a unique contribution to student school engagement, which is an important consideration in the context of supporting the academic achievement of this population because of the theorized connection between engagement and academic performance.

Findings in relation to GSA characteristics suggest that it is not enough for schools to just establish GSAs and then believe their work is done — school personnel have a responsibility after a GSA is created to nurture and maintain a culture that supports such an organization and provides students (straight/cisgender or LGBTQ) with the ability to develop activities relevant to the particular school. Efforts need to be made to encourage positive support for the GSA, countering any homophobic or transphobic communication that attempts to denigrate the group or its members. Straight and/or cisgender allies- - whether students or staff — can be trained to take the lead in creating a comprehensively supportive school climate for a GSA.

The final hypothesis was that an LGBTQ student's personal involvement in a GSA would not be a statistically significant predictor of student school engagement. This was supported by the findings. While previous research has been mixed regarding whether participation in a GSA relates to academic achievement (Hansen, 2009; Lee, 2002; Toomey et al., 2011; Walls et al., 2010), most studies have generally found more connections between GSA presence and a student's risk and resilience than between GSA membership and such outcomes (Toomey et al., 2011; Walls et al., 2013). GSA membership has more often been found to have a connection to mental health and feelings about one's sexual orientation than to fit with the school environment (Hermann, 2010; Lee, 2002; Toomey et al., 2011; Walls et 
al., 2013). Further, nothing we found has previously looked at the relationship between membership in a GSA and student school engagement, though some studies have looked at school connectedness and found a relationship to GSA attendance (Diaz et al., 2010). Findings are in line with previous research that has indicated that it is the perceptions of and context surrounding a GSA that matters for LGBTQ students, rather than whether or not they individually choose to be involved in the GSA.

While some LGBTQ students may be very interested and able to be active in a GSA at school, our study suggests that student school engagement hinges more on availability of safe adults and the characteristics of one's GSA than this decision to be involved in the GSA. Since our measure of student school engagement is meant to capture aspirations and productivity as well as belonging, students with high engagement would not only perceive greater "fit" at school but would also demonstrate goals and behaviors in relation to achieving academic success. Since GSAs tend to be focused on tasks such as providing social support and raising awareness about LGBTQ issues, rather than on academic activities (Griffin, Lee, Waugh, \& Beyer, 2004), there may not be as direct a connection between involvement and the aspirations and productivity subdomains of engagement.

The lack of significant relationship between GSA involvement and engagement supports the idea of allowing LGBTQ students to choose for themselves whether or not they wish to be involved in a GSA — involvement might not be a safe option for some students if this is perceived as "outing" themselves to the school. There is thus an important role to be played by school staff in supporting straight and/or cisgender students who wish to take on an active role of "ally" within the school setting. In situations where the school climate is particularly hostile, encouraging "ally" students to take on leadership roles within a GSA may contribute to improving the climate for LGBTQ students in the school who are not active in the GSA due to risks to their safety. 


\subsection{Limitations, Conclusions and Future Directions}

Although the present study addresses a number of gaps in the literature in relation to the connection between safe adults, GSA characteristics, and student school engagement, there are a number of limitations worth noting. First, this study utilized a convenience sample of LGBTQ youth recruited through LGBTQ organizations in a predominantly urban area in Colorado, which impacts our ability to generalize findings. In particular, our findings might not match the experiences of LGBTQ youth in other parts of the U.S. or other countries, those who live in rural areas, or those who do not attend events or seek services from LGBTQ community organizations. Second, the sample sizes for our models $(N=152$ for the full analysis, and $N=91$ for students with a GSA) were smaller than desirable and may have limited our statistical power to a point where it was difficult to detect differences in student school engagement between demographic subgroups. We did not include sexual orientation as one of our demographic variables due to the high degree of multicollinearity with gender; however, future researchers may be interested in comparing school engagement across sexual orientation subgroups. While the original dataset included high school students as well as middle school and college students, we limited our analyses to high school students due to likely developmental differences in engagement across these age groups as well as differences in these three school settings in terms of the impact of a GSA.

This study was a survey conducted at one point in time. Thus, we cannot say with certainty that the presence of safe adults at school or certain characteristics of GSAs cause greater engagement among LGBTQ students. Data were self-reported, and therefore we relied upon students' own assessment of their engagement and characteristics of GSAs; findings might differ if such constructs were assessed by teachers or others in the school system. As the survey used in this study was developed prior to Hazel et al.'s (2013) completed work validating the SSEM, some items used in the present study did not load onto 
the three domains (aspirations, belonging, productivity) in Hazel et al.'s analyses, which may impact the validity of our method of measuring student school engagement.

Additionally, the data set did not include information about student's high schools, such as the high school name, school size, average socioeconomic status of the student body, school racial demographics, or whether the school was public or private. Such school-level factors likely play key roles in student school engagement for LGBTQ students and would allow a more nuanced multi-level analysis. Additionally, while we did not collect information about high school names, we know that over half (56.6\%) of the sample currently lived closest to Denver, and so it is possible that some of the youth attend the same schools, creating some nested data. However, the size of the sample and lack of information about school(s) attended prohibited using multi-level models for these analyses. The GSA characteristics variable was a composite score due to issues with multicollinearity when including each dimension separately in the model; this prevented examining the unique contributions of each GSA characteristic (size, visibility, etc.). Further, we did not have information about how recently a GSA was established at school or the degree to which it was student-led, both of which may relate to student decisions about whether to be involved.

While we were able to answer the research questions proposed, the model looking at safe adults and GSA presence explains only $5 \%$ of the variability in student school engagement, and the model looking at GSA characteristics and GSA involvement explains only $9 \%$ of the variability, indicating small effect sizes. Other factors, such as acceptance or rejection by peers, victimization at school, school/classroom size, student involvement in school policy formation, and availability of social supports for confronting discrimination (especially among racial and ethnic minority students) likely also play a role in level of student school engagement (Fredricks et al., 2004). Exploration of these factors would provide more evidence to guide interventions to support LGBTQ youth and young adults. 
Future researchers could improve and build upon this research by replicating this study with a larger sample, with students in more rural areas, or in other regions of the country. Additionally, one might examine not just the number of types of safe adults, but the total number of safe adults that each student can access. Researchers are encouraged to measure GSA characteristics more precisely and consistently across domains by including the same response options for each dimension (size, visibility, etc.) and measuring GSA size by descriptors (i.e., small, medium, large) rather than number of students involved. The knowledge base could benefit from studies that break down these various GSA characteristics and look at the unique contributions of each to student school engagement. Additionally, this study did not explore the specific functions and activities of GSAs (e.g., support, advocacy, etc.) as perceived by LGBTQ students, and such activities may have an influence on these students' experiences and engagement at school.

Suggested future research directions include examining whether GSA characteristics predict likelihood of harassment in school, student GPA, or psychosocial outcomes such as depression. Another important direction would be to use structural equation modeling to examine whether student school engagement mediates the relationship between school contextual factors (supportive climate) and academic outcomes for LGBTQ students, as well as multi-level modelling to better understand the contribution of different levels of variables (e.g., school level, GSA level, individual level) to student school engagement for LGBTQ students. Future researchers using the SSEM are encouraged to use the latest version of the scale that has been psychometrically tested by Hazel et al. (2013), which would allow for looking more precisely at the role of each of the three domains of aspirations, belonging, and productivity for LGBTQ students.

In conclusion, this study intended to use a sample of LGBTQ high school students from one region of the U.S to examine whether safe adults at school, GSA presence, 
characteristics of GSAs, and one's personal involvement in a GSA predict student school engagement. Results indicate that presence of a greater number of types of safe adults at school predict higher student school engagement. GSA presence did not significantly relate to school engagement. Additionally, the larger, more active, more visible, and more supported a GSA is perceived to be, the more LGBTQ high school students were predicted to be engaged. As hypothesized, whether or not a student personally chose to be involved in the school's GSA did not predict student school engagement.

School-based practitioners can take concrete steps to support LGBTQ students based upon these findings, such as ensuring that school staff are trained to support these students and that the school culture supports and nurtures development of a strong and effective GSA. Staff are encouraged to allow LGBTQ students to choose for themselves whether to be involved in a GSA, with the recognition that just knowing that there is a supported GSA within the school can be more helpful for some LGBTQ students than actually being personally involved. Future research on this topic can replicate this study and work to examine additional individual and school-level factors that are related to student school engagement among LGBTQ youth.

\section{Acknowledgements}

We would like to acknowledge and thank Corey Barrett, Moises Muñoz, numerous social work interns and program volunteers, and all of the youth and young adults from Rainbow Alley who took the time to complete this survey. 


\section{References}

Birkett, M., Russell, S. T., \& Corliss, H. (2014). Sexual-orientation disparities in school: The mediational role of indicators of victimization in achievement and truancy because of feeling unsafe. American Journal of Public Health, 104(6), 1124-1128. doi:10.2105/AJPH.2013.301785

Centers for Disease Control and Prevention. (2007). YRBS Questionnaires. Retrieved from http://www.cdc.gov/healthyyouth/yrbs/questionnaire_rationale.htm

Chesir-Teran, D. (2003). Conceptualizing and assessing heterosexism in high schools: a setting-level approach. American Journal of Community Psychology, 31(3-4), 267-79. doi:10.1023/A:1023910820994

Chesir-Teran, D., \& Hughes, D. (2009). Heterosexism in high school and victimization among lesbian, gay, bisexual, and questioning students. Journal of Youth and Adolescence, 38(7), 963-75. doi:10.1007/s10964-008-9364-х

Diaz, E. M., Kosciw, J. G., \& Greytak, E. A. (2010). School connectedness for lesbian, gay, bisexual, and transgender youth: In-school victimization and institutional supports. The Prevention Researcher, 17(3), 15-18.

Estell, D. B., \& Perdue, N. H. (2013). Social support and behavioral and affective school engagement: The effects of peers, parents, and teachers. Psychology in the Schools, 50(4), 325-339. doi:10.1002/pits.21681

Fischer, S. N. (2011). School-based supports for LGBT and other minority youth: Understanding the roles of teachers, staff, gay-straight alliances, and antiharassment policies (Unpublished doctoral dissertation). New York University, New York City, NY. 
Fredricks, J. A, Blumenfeld, P. C., \& Paris, A. H. (2004). School engagement: Potential of the concept, state of the evidence. Review of Educational Research, 74(1), 59-109. doi:10.3102/00346543074001059

Garcia-Alonso, P. M. (2004). From surviving to thriving: An investigation of the utility of support groups designed to address the special needs of sexual minority youth in public high schools (Unpublished doctoral dissertation). Loyola University, Chicago, IL.

Goodenow, C., Szalacha, L., \& Westheimer, K. (2006). School support groups, other school factors, and the safety of sexual minority adolescents. Psychology in the Schools, 43(5), 573-589. doi:10.1002/pits.20173

Graybill, E. (2011). Social justice advocacy trends related to gay/straight alliance advisors' experiences in schools (Unpublished doctoral dissertation). Georgia State University, Atlanta, GA.

Greytak, E. A., Kosciw, J. G., \& Boesen, M. J. (2013). Putting the "T" in "resource": The benefits of LGBT-related school resources for transgender youth. Journal of LGBT Youth, 10(1-2), 45-63. doi:10.1080/19361653.2012.718522

Griffin, P., Lee, C., Waugh, J., \& Beyer, C. (2004). Describing roles that Gay-Straight Alliances play in schools : From individual support to school change. Journal of Gay \& Lesbian Issues in Education, 1(3), 7-22. doi:10.1300/J367v01n03_03

Hansen, A. (2009). LGBT students and allies participating in a school-based support program: School performance, connectedness, and perceptions of school climate (Unpublished doctoral dissertation). University of Minnesota, Minneapolis, MN.

Hazel, C. E., Vazirabadi, G. E., \& Gallagher, J. (2013). Measuring aspirations, belonging, and productivity in secondary students: Validation of the student school engagement measure. Psychology in the Schools, 50(7). doi:10.1002/pits 
Hazel, C. E., Vazirabadi, G. E., Albanes, J., \& Gallagher, J. (2014). Evidence of convergent and discriminant validity of the Student School Engagement Measure. Psychological Assessment, 26(3), 806-14. doi:10.1037/a0036277

Heck, N. C. (2014). Expanding, refining, and replicating research on high school gaystraight student alliances and sexual minority youth (Unpublished doctoral dissertation). University of Montana, Missoula, MT.

Heck, N. C., \& Flentje, A., \& Cochran, B. N. (2013). Offsetting risks: High school gaystraight alliances and lesbian, gay, bisexual, and transgender (LGBT) youth. Psychology of Sexual Orientation and Gender Diversity, 1, 81-90. doi: 10.1037/23290382.1.s.81

Heck, N. C., Lindquist, L. M., Stewart, B. T., Brennan, C., \& Cochran, B. N. (2013). To join or not to join: Gay-straight student alliances and the high school experiences of lesbian, gay, bisexual, and transgender youths. Journal of Gay \& Lesbian Social Services, 25(1), 77-101. doi:10.1080/10538720.2012.751764

Hermann, N. A. (2010). A correlational study of gay-straight alliances and internalized homophobia (Unpublished doctoral dissertation). Alliant International University, San Francisco, CA.

Holmes, S. E., \& Cahill, S. (2004). School experiences of gay, lesbian, bisexual, and transgender youth. Journal of Gay \& Lesbian Issues in Education, 1(3), 53-66. doi:10.1300/J367v01n03_06

Institute of Medicine. (2011). The health of lesbian, gay, bisexual and transgender people. Retrieved from http://www.ncbi.nlm.nih.gov/books/NBK64806/

Konishi, C., Saewyc, E., Homma, Y., \& Poon, C. (2013). Population-level evaluation of school-based interventions to prevent problem substance use among gay, lesbian, and 
bisexual adolescents in Canada. Preventive Medicine: An International Journal Devoted to Practice and Theory, 57, 929-933. doi: 10.1016/j.ypmed.2013.06.031

Kosciw, J., Diaz, E., \& Greytak, E. (2008). The 2007 National School Climate Survey. Retrieved from the Gay, Lesbian and Straight Education Network website: http://glsen.org/learn/research/nscs-archive

Kosciw, J. G., Greytak, E. A., Bartkiewicz, M. J., Boesen, M. J., \& Palmer, N. A. (2012). The 2011 National School Climate Survey: The experiences of lesbian, gay, bisexual and transgender youth in our nation's schools. Retrieved from the Gay, Lesbian and Straight Education Network website: http://www.glsen.org/learn/research/national

Kosciw, J. G., Greytak, E. A., Bartkiewicz, M. J., Boesen, M. J., \& Palmer, N. A. (2014). The 2013 National School Climate Survey: The experiences of lesbian, gay, bisexual, and transgender youth in our nation's schools. Retrieved from the Gay, Lesbian and Straight Education Network website: http://www.glsen.org/learn/research/national Kosciw, J. G., Greytak, E. A, \& Diaz, E. M. (2009). Who, what, where, when, and why: Demographic and ecological factors contributing to hostile school climate for lesbian, gay, bisexual, and transgender youth. Journal of Youth and Adolescence, 38(7), 976-88. doi:10.1007/s10964-009-9412-1

Kosciw, J. G., Palmer, N. A., Kull, R. M., \& Greytak, E. A. (2013). The effect of negative school climate on academic outcomes for LGBT youth and the role of in-school supports. Journal of School Violence, 12(1), 45-63. doi:10.1080/15388220.2012.732546

Lam, S., Jimerson, S., Wong, B. P. H., Kikas, E., Shin, H., Veiga, F. H., ... Zollneritsch, J. (2014). Understanding and measuring student engagement in school: The results of an international study from 12 countries. School Psychology Quarterly, 29(2), 213-232. doi:10.1037/spq0000057 
Lee, C. (2002). The impact of belonging to a high school gay/straight alliance (Unpublished doctoral dissertation). University of Utah, Salt Lake City, UT.

Li, Y., Zhang, W., Liu, J., Arbeit, M. R., Schwartz, S. J., Bowers, E. P., \& Lerner, R. M. (2011). The role of school engagement in preventing adolescent delinquency and substance use: a survival analysis. Journal of Adolescence, 34(6), 1181-92. doi:10.1016/j.adolescence.2011.07.003

Mayo, J. B., Jr. (2008). Obscene associations: Gay-straight alliances, the Equal Access Act, and abstinence-only policy. Sexuality Research \& Social Policy, 5, 45-55. doi: 10.1525/srsp.2008.5.2.45

McGuire, J. K., Anderson, C. R., Toomey, R. B., \& Russell, S. T. (2010). School climate for transgender youth: A mixed method investigation of student experiences and school responses. Journal of Youth and Adolescence, 39, 1175-1188. doi:10.1007/s10964-0109540-7

Murdock, T. B., \& Bolch, M. B. (2005). Risk and protective factors for poor school adjustment in lesbian, gay, and bisexual (LGB) high school youth: Variable and personcentered analyses. Psychology in the Schools, 42(2), 159-172. doi:10.1002/pits.20054

Murphy, H. E. (2012). Improving the lives of students, gay and straight alike: Gay-Straight Alliances and the role of school psychologists. Psychology in the Schools, 49(9), 883892. doi:10.1002/pits. 21643

Pearson, J., Muller, C., \& Wilkinson, L. (2007). Adolescent same-sex attraction and academic outcomes: The role of school attachment and engagement. Social Problems, 54(4), 523-542. doi:10.1525/sp.2007.54.4.523.524

Poteat, V. P., Sinclair, K. O., DiGiovanni, C. D., Koenig, B. A., \& Russell, S. T. (2013). Gaystraight alliances are associated with student health: A multischool comparison of 
LGBTQ and heterosexual youth. Journal of Research on Adolesence, 23, 319-330. doi: 10.1111/j.1532-7795.2012.00832.x

Poteat, V. P., Yoshikawa, H.,Calzo, J. P., Gray, M. L., DiGiovanni, C.D., Lipkin, A., MundyShephard, A., Perroti, J., Scheer, J. R., \& Shaw, M. P. (2014). Contextualizing gaystraight alliances: Student, advisor, and structural factors related to positive youth development among members. Child Development. Advanced online publication. doi: 10.1111/cdev.12289

Quasha, S. (2011). A program review of a middle school gay-straight alliance (GSA) club (Unpublished doctoral dissertation). St. Johns University, New York City, NY.

Sausa, L. A. (2005). Translating research into practice: Trans youth recommendations for improving school systems. Journal of LGBT Youth, 3(1), 15-29. doi:10.1300/J367v03n01

Seelman, K. L. (2013, October). Heterosexism and cisgenderism in high schools: Tackling the problem at multiple levels. Workshop presented at the Carolina Conference for Queer Youth, Charlotte, NC.

Seelman, K. L., Walls, N. E., Hazel, C., \& Wisneski, H. (2012). Student school engagement among sexual minority students: Understanding the contributors to predicting academic outcomes. Journal of Social Service Research, 38(1), 3-17.

doi:10.1080/01488376.2011.583829

St. John, A., Travers, R., Munro, L., Liboro, R., Schneider, M., \& Greig, C. L. (2014). The success of gay-straight alliances in Waterloo region, Ontario: A confluence of political and social factors. Journal of LGBT Youth, 11, 150-170. doi: $10.1080 / 19361653.2014 .878564$ 
Tamayo, M. (2008). The impact of high school gay, lesbian, bisexual, and transgender (GLBT) support services on the attitudes of college students in their first two years (Unpublished doctoral dissertation). Florida Atlantic University, Boca Raton, FL.

Toomey, R. B., McGuire, J. K., \& Russell, S. T. (2012). Heteronormativity, school climates, and perceived safety for gender nonconforming peers. Journal of Adolescence, 35, 187-196. doi: 10.1016/j.adolescence.2011.03.001

Toomey, R. B., Ryan, C., Diaz, R. M., \& Russell, S. T. (2011). High school Gay-Straight Alliances (GSAs) and young adult well-being: An examination of GSA presence, participation, and perceived effectiveness. Applied Developmental Science, 15(4), 175185. doi:10.1080/10888691.2011.607378

Walls, N. E., Freedenthal, S., \& Wisneski, H. (2008). Suicidal ideation and attempts among sexual minority youth receiving social services. Social Work, 53, 21-29. doi: $10.1093 / \mathrm{sw} / 53.1 .21$

Walls, N. E., Kane, S. B., \& Wisneski, H. (2010). Gay--Straight Alliances and school experiences of sexual minority youth. Youth \& Society, 41(3), 307-332. doi:10.1177/0044118X09334957

Walls, N. E., Wisneski, H., \& Kane, S. B. (2013). School climate, individual support, or both? Gay straight alliances and the mental health of sexual minority youth. School Social Work Journal, 37, 88-111.

Wang, M. T., \& Fredricks, J. A. (2014). The reciprocal links between school engagement, youth problem behaviors, and school dropout during adolescence. Child Development, 85(2), 722-37. doi:10.1111/cdev.12138

Wang, M. T., \& Peck, S. C. (2013). Adolescent educational success and mental health vary across school engagement profiles. Developmental Psychology, 49(7), 1266-76. doi:10.1037/a0030028 